\title{
Extension of Incremental Linear Discriminant Analysis to Online Feature Extraction under Nonstationary Environments
}

\author{
Annie anak Joseph ${ }^{1}$, Young-Min Jang ${ }^{2}$, Seiichi Ozawa ${ }^{1}$, and Minho Lee ${ }^{2}$ \\ 1 Graduate School of Engineering, Kobe University, \\ 1-1 Rokko-dai, Nada-ku, Kobe 657-8501, Japan \\ 097t805t@stu.kobe-u.ac.jp, ozawasei@kobe-u.ac.jp \\ 2 Electrical and Electronic Engineering, Kyungpook National University \\ 1370 Sangyeok-dong, Buk-gu, Taegu 702-701, Korea \\ ymjang@ee.knu.ac.kr, mholee@knu.ac.kr
}

\begin{abstract}
In this paper, a new approach to an online feature extraction under nonstationary environments is proposed by extending Incremental Linear Discriminant Analysis (ILDA). The extended ILDA not only detect so-called "concept drifts" but also transfer the knowledge on discriminant feature spaces of the past concepts to construct good feature spaces. The performance of the extended ILDA is evaluated for the benchmark datasets including sudden changes and reoccurrence in concepts.
\end{abstract}

Keywords: incremental learning, concept drift, online feature extraction, linear discriminant analysis, knowledge transfer.

\section{Introduction}

Recently, online incremental learning becomes popular and increasingly important due to the wide range of online applications such as person identification and pattern recognition [12. However, in the real world, the learning is often enforced under nonstationary environments where a target function or a class boundary changes over time, resulting in increasing loss of relevance between the current and the previous concepts. This leads to the need of model changes to adapt to the current concept promptly 3 . This dynamic nature in actual environments is called "concept drifts" [3]. And it is expected that the performance of the current model would be seriously dropped unless the model has ability to detect the concept drift and to update itself by adapting to changing environments.

Previous types of incremental feature extraction such as Incremental Principal Component Analysis (IPCA) [1] and Incremental Linear Discriminant Analysis (ILDA) 214 do not provide this ability. However, we believe that the detection of concept drifts for the feature extraction purposes also plays an important role to enhance the system performance. In order to extract good features under 